\title{
Blow-up criteria for the inhomogeneous nonlinear Schrödinger equation
}

Han Yang ${ }^{1}$ and Shihui Zhu ${ }^{2 *}$

\section{"Correspondence:}

shihuizhumath@gmail.com

${ }^{2}$ College of Mathematics and Software Science, Sichuan Normal University, Chengdu, 610066, China Full list of author information is available at the end of the article

\begin{abstract}
In this paper, using the variational characteristic of the virial identity and a new estimate of the kinetic energy, we obtain a new sufficient condition for the existence of blow-up solutions.

MSC: 35Q55; 35B44

Keywords: inhomogeneous nonlinear Schrödinger equation; blow-up criteria; virial identity
\end{abstract}

\section{Introduction}

In this paper, we study the Cauchy problem of the inhomogeneous nonlinear Schrödinger equation (INSE)

$$
\begin{aligned}
& i u_{t}+\Delta u+|x|^{b}|u|^{p-2} u=0, \quad t \geq 0, x \in \mathbb{R}^{N}, \\
& u(0, x)=u_{0},
\end{aligned}
$$

where $i^{2}=-1 ; \triangle=\sum_{j=1}^{N} \frac{\partial^{2}}{\partial x_{j}^{2}}$ is the Laplace operator in $\mathbb{R}^{N} ; u=u(t, x):[0, T) \times \mathbb{R}^{N} \rightarrow \mathbb{C}$ is the complex valued function and $0<T \leq+\infty$; the parameter $b \geq 0$ and $2<p<\tilde{p}$ (we use the convention: $\tilde{p}=+\infty$ for $N=2, \tilde{p}=\frac{2 N}{N-1}+\frac{2 b}{N-1}$ for $N \geq 3$ ); $N \geq 2$ is the space dimension. A few years ago, it was suggested that stable high power propagation can be achieved in a plasma by sending a preliminary laser beam that creates a channel with a reduced electron density, and thus reduces the nonlinearity inside the channel (see $[1,2])$. In this case, the beam propagation can be modeled by the inhomogeneous nonlinear Schrödinger equation in the following form:

$$
i \phi_{t}+\triangle \phi+K(x)|\phi|^{p-2} \phi=0, \quad \phi(0, x)=\varphi \in H^{1}\left(\mathbb{R}^{N}\right) .
$$

Recently, this type of inhomogeneous nonlinear Schrödinger equations has been widely investigated. When $k_{1} \leq K(x) \leq k_{2}$ with $k_{1}, k_{2}>0$ and $p=2+\frac{4}{N}$, Merle [3] proved the existence and nonexistence of blow-up solutions to the Cauchy problem (1.3). When $K(x)=K(\varepsilon|x|) \in C^{4}\left(\mathbb{R}^{N}\right) \cap L^{\infty}\left(\mathbb{R}^{N}\right)$ with small $\varepsilon$ and $p=2+\frac{4}{N}$, Fibich, Liu and Wang [2, 4] obtained the stability and instability of standing waves to the Cauchy problem (1.3).

\section{Springer}

(02014 Yang and Zhu; licensee Springer. This is an Open Access article distributed under the terms of the Creative Commons Attribution License (http://creativecommons.org/licenses/by/2.0), which permits unrestricted use, distribution, and reproduction in any medium, provided the original work is properly cited. 
We recall some known results on the blow-up solutions for the classical nonlinear Schrödinger equation

$$
i v_{t}+\Delta v+|v|^{p-2} v=0, \quad v(0, x)=v_{0} \in H^{1}\left(\mathbb{R}^{N}\right) .
$$

Ginibre and Velo [5] showed the local well-posedness in $H^{1}\left(\mathbb{R}^{N}\right)$. Glassey [6] showed the existence of blow-up solutions when the energy is negative and $|x| v_{0} \in L^{2}\left(\mathbb{R}^{N}\right)$. Ogawa and Tsutsumi [7] obtained the existence of blow-up solutions in radial case without the restriction $|x| v_{0} \in L^{2}\left(\mathbb{R}^{N}\right)$. Weinstein [8] and Zhang [9] obtained the sharp conditions of global existence for critical and supercritical nonlinearity. Merle and Raphaël [10] showed the existence of blow-up solutions without $|x| v_{0} \in L^{2}\left(\mathbb{R}^{N}\right)$ for $p=2+\frac{4}{N}$. Lushnikov [11] and Holmer et al. [12] obtained some sufficient conditions for existence of blow-up for $p=4$ and $N=3$ basing on an estimate of the kinetic energy.

In this paper, we study blow-up criteria for the Cauchy problem (1.1)-(1.2) with $p>$ $\frac{2 N+2 b+4}{N}$, where the nonlinearity $|x|^{b}|u|^{p-2} u$ includes an unbounded potential $|x|^{b}$. We note that (1.1) has scaling: $u_{\lambda}(t, x)=\lambda^{\frac{2+b}{p-2}} u\left(\lambda^{2} t, \lambda x\right)$ is a solution if $u(t, x)$ is a solution. The

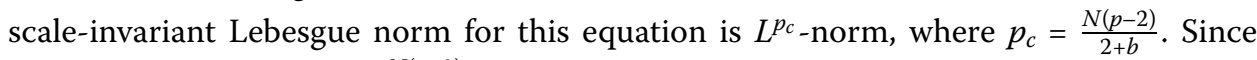
$p>\frac{2 N+2 b+4}{N}$, we have $p_{c}=\frac{N(p-2)}{2+b}>2$ and we may call (1.1) a class of Schrödinger equations with $L^{2}$-super critical nonlinearity. Chen and Guo [13] and Chen [14] showed the local well-posedness of the Cauchy problem (1.1)-(1.2) in $H_{r}^{1}=H_{r}^{1}\left(\mathbb{R}^{N}\right)$, where $H_{r}^{1}\left(\mathbb{R}^{N}\right)$ is the set of radially symmetric functions in $H^{1}\left(\mathbb{R}^{N}\right)$. Moreover, $u(t, x)$ satisfies the following conservation laws:

$$
\int_{\mathbb{R}^{N}}|u(t, x)|^{2} d x=\int_{\mathbb{R}^{N}}\left|u_{0}\right|^{2} d x
$$

and

$$
E\left(u_{0}\right)=E(u):=\frac{1}{2} \int_{\mathbb{R}^{N}}|\nabla u|^{2} d x-\frac{1}{p} \int_{\mathbb{R}^{N}}|x|^{b}|u|^{p} d x .
$$

Chen and Guo [13] also showed the sharp conditions of blow-up and global existence of solutions to the Cauchy problem (1.1)-(1.2) by the cross-constrained variational arguments. On the other hand, letting $J(t):=\int_{\mathbb{R}^{N}}|x|^{2}|u(t, x)|^{2} d x$, this can be interpreted as the average width of the initial distribution $|u|$. It follows from Chen and Guo's results in [13] that we have the following proposition.

Proposition 1.1 Assume that $u_{0} \in H_{r}^{1},|x| u_{0} \in L^{2}$ and the corresponding solution $u(t, x)$ of the Cauchy problem (1.1)-(1.2) on the interval $[0, T)$. Then, for all $t \in[0, T)$, one has $J(t):=\int|x|^{2}|u(t, x)|^{2} d x<+\infty$,

$$
J^{\prime}(t)=4 \Im \int_{\mathbb{R}^{N}} x \bar{u} \nabla u d x
$$

and

$$
J^{\prime \prime}(t)=4(N p-2 N-2 b) E\left(u_{0}\right)-2(N p-2 N-2 b-4) \int_{\mathbb{R}^{N}}|\nabla u|^{2} d x .
$$


In the case $N p-2 N-2 b-4 \geq 0$, it follows from the last relation that

$$
J^{\prime \prime}(t) \leq 4(N p-2 N-2 b) E\left(u_{0}\right)
$$

and for $E\left(u_{0}\right)<0$ the positive-definite quantity $J(t)$ becomes negative over a finite time by virtue of the above inequality. This means that a singularity appears in the solution of the given INSE. Indeed, applying Weinstein's arguments [8] and the classical analysis identity

$$
J(t)=J(0)+J^{\prime}(0) t+\int_{0}^{t} J^{\prime \prime}(t-s) s d s
$$

one has the following theorem (see also Chen and Guo [13]).

Theorem 1.2 Let $N \geq 2,0<b<N-2$ and $\frac{2 N+2 b+4}{N}<p<\tilde{p}$ (where $\tilde{p}=+\infty$ for $N=2$, $\tilde{p}=\frac{2 N}{N-2}+\frac{2 b}{N-1}$ for $\left.N \geq 3\right)$. Assume $u_{0} \in H^{1}\left(\mathbb{R}^{N}\right)$ and $|x| u_{0} \in L^{2}\left(\mathbb{R}^{N}\right)$ is radially symmetric. If the initial data satisfies either

(i)

$$
E\left(u_{0}\right)<0
$$

(ii)

$$
E\left(u_{0}\right)=0 \text { and } J^{\prime}(0)<0 \text {, }
$$

(iii) $E\left(u_{0}\right)>0$ and

$$
J^{\prime}(0)<-2 \sqrt{2(N p-2 N-2 b) E\left(u_{0}\right)} J(0),
$$

then there exists $0<T<+\infty$ such that the corresponding solution $u(t, x)$ blows up in finite time $T$.

We remark that in the case $E\left(u_{0}\right)>0, N p-2 N-2 b-4 \geq 0$ both collapse and spreading of the initial disturbance are possible. Although the INSE is no longer applicable near the formation point of a singularity and dissipative or some other limiting mechanism come to play. It is very important to be able to predict the presence or absence of collapse for different classes of initial conditions. The sufficient conditions for existence of blowup solutions are given in [13] if either $E\left(u_{0}\right)<0$ or $J^{\prime}(0)<0$. A natural question arises whether there is a sufficient condition for existence of blow-up solutions with $E\left(u_{0}\right)>0$ and $J^{\prime}(0)>0$.

In the present paper, motivated by the studies of the classical nonlinear Schrödinger equation (see $[8,11,12])$, we use variational characteristic of second-order derivatives of the virial identity to catch up with the information of $\|\nabla u\|_{L^{2}}$, and we obtain a new sufficient condition for the existence of blow-up solutions to the inhomogeneous nonlinear 
Schrödinger equation (1.1). More precisely, let

$$
g(y)= \begin{cases}\sqrt{y+\frac{4}{N p-2 b-2 N-4} y^{-\frac{N p-2 b-2 N-4}{4}}-\frac{N p-2 b-2 N}{N p-2 b-2 N-4}} & \text { if } 0<y<1, \\ -\sqrt{y+\frac{4}{N p-2 b-2 N-4} y^{-\frac{N p-2 b-2 N-4}{4}}-\frac{N p-2 b-2 N}{N p-2 b-2 N-4}} & \text { if } y \geq 1 .\end{cases}
$$

Then we have the following theorem.

Theorem 1.3 Let $N \geq 2,0<b<N-2$ and $\frac{2 N+2 b+4}{N}<p<\min \left\{\frac{2 b+2 N+12}{N}\right.$, $\left.\tilde{p}\right\}$. Assume that $u_{0} \in H^{1}\left(\mathbb{R}^{N}\right)$ and $|x| u_{0} \in L^{2}\left(\mathbb{R}^{N}\right)$ is radially symmetric. If

$$
J^{\prime}(0)<2 \sqrt{\frac{N p-2 b-2 N-4}{N p-2 b-2 N}} N\left\|u_{0}\right\|_{L^{2}}^{2} g\left(\frac{8(N p-2 b-2 N) E\left(u_{0}\right)}{N^{2}(N p-2 b-2 N-4)\left\|u_{0}\right\|_{L^{2}}^{4}} J(0)\right),
$$

where $g(x)$ is defined by (1.11), then there exists $0<T<+\infty$ such that the corresponding solution $u(t, x)$ blows up in finite time $T$.

\section{Notations and preliminaries}

In this paper, we denote $L^{q}\left(\mathbb{R}^{N}\right),\|\cdot\|_{L^{q}\left(\mathbb{R}^{N)}\right.}, H^{s}\left(\mathbb{R}^{N}\right)$ and $\int_{\mathbb{R}^{N}} \cdot d x$ by $L^{q},\|\cdot\|_{L^{q}}, H^{s}$ and $\int \cdot d x$, respectively. $\Re z$ and $\Im z$ are the real part and imaginary part of the complex number $z$, respectively. $\bar{z}$ is denoted the complex conjugate of the complex number $z$. The various positive constants will be simply denoted by $C$.

For the Cauchy problem (1.1)-(1.2), the space we work in is

$$
H_{r}^{1}:=\left\{u \in H^{1} \mid u(x)=u(r)\right\}, \quad \text { where } r=\sqrt{x_{1}^{2}+x_{2}^{2}+\cdots+x_{N}^{2}},
$$

which is a Hilbert space. Moreover, we define the energy functional $E(u(t))$ in $H_{r}^{1}$ by

$$
E(u(t)):=\frac{1}{2} \int|\nabla u(t, x)|^{2} d x-\frac{1}{p} \int|x|^{b}|u(t, x)|^{p} d x .
$$

The functional $E(u)$ is well-defined according to the Sobolev embedding theorem (see [15]). Chen and Guo [13] and Chen [14] showed the local well-posedness for the Cauchy problem (1.1)-(1.2) in $H_{r}^{1}$, as follows.

Proposition 2.1 Let $N \geq 2, b \geq 0$ and $2+2 b /(N-1)<p<\tilde{p}$ (where $\tilde{p}=+\infty$ for $N=2, \tilde{p}=$ $\frac{2 N}{N-2}+\frac{2 b}{N-1}$ for $\left.N \geq 3\right)$. For any $u_{0} \in H_{r}^{1}$, there exists a unique solution $u(t, x)$ of the Cauchy problem (1.1)-(1.2) on the maximal time interval $[0, T)$ such that $u(t, x) \in C\left([0, T) ; H_{r}^{1}\right)$ and either $T=+\infty$ (global existence), or $T<+\infty$ and $\lim _{t \rightarrow T}\|u(t, x)\|_{H_{r}^{1}}=+\infty($ blow-up $)$. Furthermore, for all $t \in[0, T), u(t, x)$ satisfies the following conservation laws:

(i) Conservation of mass: $\|u(t)\|_{2}=\left\|u_{0}\right\|_{2}$.

(ii) Conservation of energy: $E(u(t, x))=E\left(u_{0}\right)$.

In addition, by some basic calculations, we have the following lemma, which gives further insight in the dynamic criterion for collapse proposed by Lushnikov in [11].

Lemma 2.2 If $V(t)>0$ is the positive solution of the following differential equation:

$$
\frac{d^{2}}{d t^{2}} V=f(V)
$$


and there exists $0<T_{1}<+\infty$ such that $\lim _{t \rightarrow T_{1}} V(t)=0$, then for the solution $\tilde{V}>0$ of the following differential equation:

$$
\tilde{V}_{t t}=f(\tilde{V})-h^{2}(t) \leq f(\tilde{V})
$$

there exists $0<T_{2} \leq T_{1}<+\infty$ such that $\lim _{t \rightarrow T_{2}} \tilde{V}(t)=0$.

Proof Since the function $-h^{2}(t)$ is non-positive, which pulls $\tilde{V}(t)$ to zero more quickly than $V(t)$ (see also [11]), one sees that the conclusion in Lemma 2.2 is true by the classical analysis identity (1.7).

\section{Proof of Theorem 1.3}

Since $|x| u_{0} \in L^{2}$, we have $|x| u(t, x) \in L^{2}$ by the local well-posedness. Taking $J:=J(t)=$ $\int|x|^{2}|u|^{2} d x$, by Proposition 1.1, we get $J^{\prime}(t)=4 \Im \int x \cdot \nabla u \bar{u} d x$ and

$$
J^{\prime \prime}(t)=4(N p-2 N-2 b) E\left(u_{0}\right)-2(N p-2 N-2 b-4) \int|\nabla u|^{2} d x .
$$

It follows from some calculations that

$$
\int|u|^{2} d x=\frac{1}{N} \int \nabla \cdot x|u|^{2} d x=-\frac{2}{N} \Re \int x \cdot \nabla u \bar{u} d x .
$$

Then we get $\frac{N}{2}\|u\|_{L^{2}}^{2} \leq\|x u\|_{L^{2}}\|\nabla u\|_{L^{2}}$, and by the fact that $|z|^{2}=|\Re z|^{2}+|\Im z z|^{2}$ we deduce that

$$
\begin{aligned}
\|\nabla u\|_{L^{2}}^{2}\|x u\|_{L^{2}}^{2} & \geq\left|\int x \cdot \nabla u \bar{u} d x\right|^{2} \\
& =\frac{N^{2}}{4}\|u\|_{L^{2}}^{4}+\left|\Im \int x \cdot \nabla u \bar{u} d x\right|^{2}=\frac{N^{2}}{4}\|u\|_{L^{2}}^{4}+\frac{\left|J^{\prime}(t)\right|^{2}}{16} .
\end{aligned}
$$

Injecting (3.2) into (3.1), by the conservation laws, we deduce that

$$
\begin{aligned}
J^{\prime \prime}(t) \leq & 4(N p-2 N-2 b) E\left(u_{0}\right) \\
& -\frac{N^{2}[N p-2 N-2 b-4]}{2 J}\left\|u_{0}\right\|_{L^{2}}^{4}-\frac{N p-2 N-2 b-4}{8 J} J_{t}^{2} .
\end{aligned}
$$

Letting $J=A^{\frac{8}{N p-2 N-2 b+4}}$ and rewriting (3.3) to remove the last term with $J_{t}^{2}$, we have

$$
\begin{aligned}
A_{t t} \leq & \frac{(N p-2 b-2 N)(N p-2 b-2 N+4)}{2} E\left(u_{0}\right) A^{\frac{N p-2 b-2 N-4}{N p-2 b-2 N+4}} \\
& -\frac{N^{2}(N p-2 b-2 N-4)(N p-2 b-2 N+4)}{16}\left\|u_{0}\right\|_{L^{2}}^{4} A^{-\frac{12+2 b+2 N-N p}{N p-2 b-2 N+4}},
\end{aligned}
$$

which has a simple mechanism analogy. Multiplying $A_{t}$ in (3.4) and integrating with the time variable $t$, we get the corresponding mechanical energy

$$
\varepsilon(t)=\frac{1}{2} A_{t}^{2}+U(A(t))
$$


where

$$
\begin{aligned}
U(A)= & -\frac{(N p-2 b-2 N+4)^{2}}{4} E\left(u_{0}\right) A^{\frac{2(N p-2 b-2 N)}{N p-2 b-2 N+4}} \\
& +\frac{N^{2}(N p-2 b-2 N+4)^{2}}{32}\left\|u_{0}\right\|_{L^{2}}^{4} A^{\frac{2(N p-2 b-2 N-4)}{N p-2 b-2 N+4}} .
\end{aligned}
$$

Restricting ourselves to the case $E>0$, and according to the assumptions on $p, b$ and $N$ we see that $U(A)$ achieves its maximum $U_{\max }$ at $A_{\max }$ with

$$
A_{\max }=\left(\frac{N^{2}(N p-2 b-2 N-4)\left\|u_{0}\right\|_{L^{2}}^{4}}{8(N p-2 b-2 N) E\left(u_{0}\right)}\right)^{\frac{N p-2 b-2 N+4}{8}}
$$

and

$$
U_{\max }=\frac{(N p-2 b-2 N+4)^{2}}{N p-2 b-2 N-4} E\left(u_{0}\right)\left(\frac{N^{2}(N p-2 b-2 N-4)\left\|u_{0}\right\|_{L^{2}}^{4}}{8(N p-2 b-2 N) E\left(u_{0}\right)}\right)^{\frac{N p-2 b-2 N}{4}} .
$$

To facilitate the rest of the analysis, we introduce a rescaling. Define $\tilde{J}(s)$ and $\tilde{\varepsilon}(s)$ by the relations

$$
\begin{aligned}
& A(t)=A_{\max } \tilde{A}(\alpha t), \quad \varepsilon(t)=U_{\max } \tilde{\varepsilon}(\alpha t), \\
& \alpha=\sqrt{\frac{N p-2 b-2 N}{N p-2 b-2 N-4}} \frac{16 E\left(u_{0}\right)}{N\left\|u_{0}\right\|_{L^{2}}^{2}}, \quad s=\alpha t .
\end{aligned}
$$

Thus, by (3.4), $\tilde{A}$ satisfies the following differential inequality:

$$
\tilde{A}_{s s} \leq \frac{N p-2 b-2 N+4}{64}\left(\tilde{A}^{\frac{N p-2 b-2 N-4}{N p-2 b-2 N+4}}-\tilde{A}^{-\frac{12+2 b+2 N-N p}{N p-2 b-2 N+4}}\right) .
$$

Applying Lemma 2.2, if we show that for the positive solution of the following differential equation:

$$
\tilde{A}_{s S}=\frac{N p-2 b-2 N+4}{64}\left(\tilde{A}^{\frac{N p-2 b-2 N-4}{N p-2 b-2 N+4}}-\tilde{A}^{-\frac{12+2 b+2 N-N p}{N p-2 b-2 N+4}}\right),
$$

there exists a time $0<s_{1}<+\infty$ and $\lim _{s \rightarrow s_{1}} \tilde{A}(s)=0$, then for the positive solution of the differential inequality (3.6), there exists a time $0<s_{2} \leq s_{1}<+\infty$ and $\lim _{s \rightarrow s_{2}} \tilde{A}(s)=0$. Indeed, setting

$$
\tilde{U}(\tilde{A})=-\frac{N p-2 b-2 N-4}{4} \tilde{A}^{\frac{2(N p-2 b-2 N)}{N p-2 b-2 N+4}}+\frac{N p-2 b-2 N}{4} \tilde{A}^{\frac{2(N p-2 b-2 N-4)}{N p-2 b-2 N+4}},
$$

we see that (3.5) converts to

$$
\tilde{\varepsilon}(s)=\frac{16(N p-2 b-2 N-4)}{(N p-2 b-2 N+4)^{2}} \tilde{A}_{s}^{2}(s)+\tilde{U}(\tilde{A}(s)) .
$$

It is obvious that the maximum of $\tilde{U}(\tilde{A}(s))$ is 1 , which is attained by the maximum at $\tilde{A}(s)=1$. By the variational characteristic of $\tilde{U}(\tilde{A}(s))$, we claim that under one of the follow- 
ing conditions, $\tilde{A}(s)$ vanishes in finite time, so does $J(t)$ (which implies the solution $u(t, x)$ blows up in finite time):

(a) $\tilde{\varepsilon}(0)<1$ and $\tilde{A}(0)<1$,

(b) $\tilde{\varepsilon}(0) \geq 1$ and $\tilde{A}_{s}(0)<0$.

Indeed, it follows from (3.7) and (3.9) that $\tilde{\varepsilon}(s)=\tilde{\varepsilon}(0)$. (a) If $\tilde{\varepsilon}(0)<1$, then $\tilde{\varepsilon}(t)<1$ and $\tilde{U}(\tilde{A}(s))<1$ for $t \in I$ (maximal existence interval). By the assumption $\tilde{A}(0)<1$, we deduce that $\tilde{A}(s)<1$ for $t \in I$. Moreover, we have

$$
\tilde{A}^{\frac{N p-2 b-2 N-4}{N p-2 b-2 N+4}}-\tilde{A}^{-\frac{12+2 b+2 N-N p}{N p-2 b-2 N+4}}<0 .
$$

Using (3.7) and the classical analysis identity

$$
\tilde{A}(s)=\tilde{A}(0)+\tilde{A}^{\prime}(0) s+\int_{0}^{s} \tilde{A}^{\prime \prime}(s-\tau) \tau d \tau
$$

we see that $\tilde{A}(s)$ vanishes in finite time. On the other hand, for the second case (b), if $\tilde{\varepsilon}(0) \geq 1$, then $\tilde{\varepsilon}(t) \geq 1$. If follows from (3.9) that $\tilde{U}(\tilde{A}(s))=1$, which implies that $\tilde{A}(s)=1$ and

$$
\tilde{A}^{\frac{N p-2 b-2 N-4}{N p-2 b-2 N+4}}-\tilde{A}^{-\frac{12+2 b+2 N-N p}{N p-2 b-2 N+4}}=0 .
$$

Using (3.7) and the classical analysis identity (3.10), we deduce that if $\tilde{A}_{s}(0)<0$, then $\tilde{A}(s)$ vanishes in finite time. This completes the proof of claim (a) and (b). Now, we return to the proof of Theorem 1.3. If we define $\tilde{J}=\tilde{A}^{\frac{8}{N p-2 b-2 N+4}}$, then (3.9) is equal to

$$
\tilde{\varepsilon}(s)=\frac{N p-2 b-2 N-4}{4} \tilde{J}^{\frac{N p-2 b-2 N-4}{4}}\left[\tilde{J}_{s}^{2}-\tilde{J}+\frac{N p-2 b-2 N}{N p-2 b-2 N-4}\right] .
$$

Taking

$$
h(y)=\sqrt{y+\frac{4}{N p-2 b-2 N-4} y^{-\frac{N p-2 b-2 N-4}{4}}-\frac{N p-2 b-2 N}{N p-2 b-2 N-4}},
$$

we see that

$$
\tilde{\varepsilon}<1 \quad \Leftrightarrow \quad \tilde{J}_{s} \mid<h(\tilde{J}) \quad \text { and } \quad \tilde{\varepsilon} \geq 1 \quad \Leftrightarrow \quad\left|\tilde{J}_{s}\right| \geq h(\tilde{J}) .
$$

Thus condition (a) is true if and only if

$$
\tilde{J}(0)<1 \quad \text { and } \quad-h(\tilde{J}(0))<\tilde{J}_{s}(0)<h(\tilde{J}(0))
$$

and condition (b) is true if and only if $\tilde{J}_{s}(0)<-h(\tilde{J}(0))$. Collecting the above two conditions, we deduce that the solution $u(t, x)$ blows up in finite time $0<T<+\infty$ provided

$$
\tilde{J}_{s}(0)< \begin{cases}h(\tilde{J}(0)) & \text { if } 0<\tilde{J}(0)<1 \\ -h(\tilde{J}(0)) & \text { if } \tilde{J}(0) \geq 1\end{cases}
$$


Finally, substituting back $J(t)$, we see that

$$
\tilde{J}(0)=\frac{8(N p-2 b-2 N) E\left(u_{0}\right)}{N^{2}(N p-2 b-2 N-4)\left\|u_{0}\right\|_{L^{2}}^{4}} J(0), \quad \tilde{J}_{s}(0)=\sqrt{\frac{N p-2 b-2 N}{N p-2 b-2 N-4}} \frac{J_{t}(0)}{2 N\left\|u_{0}\right\|_{L^{2}}^{2}},
$$

which implies that (3.12) is equivalent to

$$
J_{t}(0)< \begin{cases}2 \sqrt{\frac{N p-2 b-2 N-4}{N p-2 b-2 N}} N\left\|u_{0}\right\|_{L^{2}}^{2} h\left(\frac{8(N p-2 b-2 N) E\left(u_{0}\right)}{N^{2}(N p-2 b-2 N-4)\left\|u_{0}\right\|_{L^{2}}^{4}} J(0)\right) & \text { if } 0<\tilde{J}(0)<1, \\ -2 \sqrt{\frac{N p-2 b-2 N-4}{N p-2 b-2 N}} N\left\|u_{0}\right\|_{L^{2}}^{2} h\left(\frac{8(N p-2 b-2 N) E\left(u_{0}\right)}{N^{2}(N p-2 b-2 N-4)\left\|u_{0}\right\|_{L^{2}}^{4}} J(0)\right) & \text { if } \tilde{J}(0) \geq 1 .\end{cases}
$$

\section{Competing interests}

The authors declare that they have no competing interests.

\section{Authors' contributions}

HY participated in the design of the study, SZ studied the virial identity, participated in the sequence alignment and drafted the manuscript. All authors read and approved the final manuscript.

\section{Author details}

${ }^{1}$ College of Mathematics, Southwest Jiaotong University, Chengdu, 610031, China. ${ }^{2}$ College of Mathematics and Software Science, Sichuan Normal University, Chengdu, 610066, China.

\section{Acknowledgements}

This work is supported partly by the National Natural Science Foundation of P.R. China grants 11226162 and 11371267.

\section{Received: 3 November 2013 Accepted: 20 January 2014 Published: 04 Feb 2014}

\section{References}

1. Gill, TS: Optical guiding of laser beam in nonuniform plasma. Pramana J. Phys. 55, 842-845 (2000)

2. Liu, CS, Tripathi, VK: Laser guiding in an axially nonuniform plasma channel. Phys. Plasmas 1, $3100-3103$ (1994)

3. Merle, F: Nonexistence of minimal blow up solutions of equations $i u_{t}=-\Delta u-K(x)|u|^{4 / N} u$ in $R^{N}$. Ann. IHP, Phys. Théor. 64, 33-85 (1996)

4. Liu, Y, Wang, XP, Wang, K: Instability of standing waves of the Schrödinger equations with inhomogeneous nonlinearities. Trans. Am. Math. Soc. 358, 2105-2122 (2006)

5. Ginibre, J, Velo, G: On a class of nonlinear Schrödinger equations. I. The Cauchy problem, general case. J. Funct. Anal. 32, 1-32 (1979)

6. Glassey, RT: On the blowing up of solutions to the Cauchy problem for nonlinear Schrödinger equations. J. Math. Phys. 18, 1794-1797 (1977)

7. Ogawa, T, Tsutsumi, Y: Blow-up of $H^{1}$ solution for the nonlinear Schrödinger equation. J. Differ. Equ. 92, 317-330 (1991)

8. Weinstein, Ml: Nonlinear Schrödinger equations and sharp interpolation estimates. Commun. Math. Phys. 87, 567-576 (1983)

9. Zhang, J: Sharp conditions of global existence for nonlinear Schrödinger and Klein-Gordon equations. Nonlinear Anal. 48, 191-207 (2002)

10. Merle, F, Raphaël, P: Blow-up dynamic and upper bound on the blow-up rate for critical nonlinear Schrödinger equation. Ann. Math. 16, 157-222 (2005)

11. Lushnikov, PM: Dynamic criterion for collapse. JETP Lett. 62, 461-467 (1995)

12. Holmer, J, Platte, R, Roudenko, S: Blow-up criteria for the 3D cubic nonlinear Schrödinger equation. Nonlinearity 23, 977-1030 (2010)

13. Chen, JQ, Guo, BL: Sharp global existence and blowing up results for inhomogeneous Schrödinger equations. Discrete Contin. Dyn. Syst., Ser. B 8, 357-367 (2007)

14. Chen, JQ: On a class of nonlinear inhomogeneous Schrödinger equation. J. Appl. Math. Comput. 32, 237-253 (2010)

15. Cazenave, T: Semilinear Schrödinger Equations. Courant Lecture Notes in Mathematics, vol. 10. Am. Math. Soc., Providence (2003)

10.1186/1029-242X-2014-55

Cite this article as: Yang and Zhu: Blow-up criteria for the inhomogeneous nonlinear Schrödinger equation. Journal of Inequalities and Applications 2014, 2014:55 